\title{
ENHANCED DAMPING CHARACTERISTICS OF TIMOSHENKO BEAM ON ELASTIC AND METAMATERIAL FOUNDATIONS
}

\author{
A. O. Oyelade*1, O. M. Sadiq ${ }^{1}$ \\ ${ }^{1}$ Department of Civil and Environmental Engineering, Faculty of Engineering, University of \\ Lagos, Akoka, Nigeria \\ e-mail: aoyelade@unilag.edu.ng; osadiq@unilag.edu.ng \\ *corresponding author
}

\begin{abstract}
An analytical model is developed for the flexural wave propagation of a continuous Timoshenko beam resting on elastic and metamaterial foundations. The metamaterial foundation consists of positive and negative springs with a damper. This added negative stiffness component is constructed in such a way to provide the same static stiffness and the same damping component with the equivalent reference beam on elastic foundation. Numerical examples are used to investigate the effect of the shear on wavenumber and damping for beam with elastic and metamaterial foundations. The effects of engineering safety, damping coefficient and resonating mass on the dissipative property of the beam is investigated analytically. The simulation results provide indication of an enhanced damping characteristics for the damping ratio of the flexural waves propagating within the beam.
\end{abstract}

Keywords: Timoshenko beams, metamaterial, flexural waves, negative stiffness

\section{Introduction}

The dynamic analysis of beams on elastic foundations is of theoretical and practical importance in many engineering fields, especially in civil engineering for soil-structure interaction problems and in railway engineering for vehicle-railway vibrations. Different foundation models and beam theories (Euler-Bernoulli beam or Rayleigh-Timoshenko beam) have been adopted and numerous research papers have been published on this topic (Haldar \& Basu 2013; Yu et al. 2006; Zhou et al. 2017). The simplest and most frequently employed theory for a continuous beam is that of Euler-Bernoulli beam which is widely used in the research community. Wang and So (Wang \& So 2015) defined the drawbacks of using Euler-Bernoulli beam theory in dealing with the deep beams. The novel model of Timoshenko is the inclusion of shear deformation forces, which were omitted in Euler-Bernoulli model. Likewise, in Euler-Bernoulli theory, the cross section was assumed constant whereas in the Timoshenko theory, shear forces make the cross section rotate (Kahrobaiyan et al. 2014).

$\mathrm{Yu}$ et al. (Yu et al. 2006) investigated the propagation properties of flexural waves in the periodic Euler beam resting on Winkler foundations. A finite element model was developed for the stability analysis of a Timoshenko beam resting on an elastic foundation under periodic axial loads (B. A. H. Abbas and J. Thomas, 1978). The influence of elastic foundation and two 
boundary conditions were considered on the Timoshenko beam. In addition, the effect of Winkler elastic foundation modulus, slenderness ratio and elastically restrained boundary conditions on the critical load of a Timoshenko beam subjected to an end follower force was investigated(Lee et al., 1992). The Recursive differentiation method was used to study the stability characteristics of axially loaded cantilever Timoshenko beam resting on two parameter foundation by Abohadima et al. (Abohadima et al. 2015). The obtained solutions were verified with published papers and good agreement was achieved. Jiang et al. (Zhang, 2019) used an alternative method, the reverberation-ray matrix method, to analysed free vibration of Timoshenko beams resting on a general elastic foundation. Sometimes, Timoshenko beam is under an initial stress, in which the dynamic behaviour is quite different from unstressed beam, hence a numerical approach was developed to understand the effect of initial stress on the attenuation zones and attenuation coefficients of periodic Timoshenko beams resting on a two parameter elastic foundation (Liu et al. 2016).

Considering damping properties as a mechanism of vibration reduction, the characteristics of externally damped beam structures have been examined in literature (Capsoni et al. 2013). In designing a continuous structures, the most regularly used extremely dissipative damping materials are made up of low to moderate stiffness. Therefore, their structure is unfit for practical application where large load carrying capacity is required (Rivin, 2010). Chronopoulos et al. (Chronopoulos et al. 2015) investigated wave dissipation in a continuous and layered periodic beam where Euler-Bernoulli model was used in the governing equation. It was discovered that a drastic rise of numerous orders of magnitude was displayed for the damping ratio of the flexural waves propagating within the modelled configurations of the beam having negative stiffness component. The suitability of the aforementioned work in predicating the practical response of the beam led the author to reconsider the derivation of flexural waves propagation in Timoshenko beam in the presence of negative stiffness component.

This paper proposes an effective approach to increase the damping features of continuous Timoshenko beam by incorporating a negative stiffness element. In Section 2, a brief description is given of the negative stiffness components and the damping connection with the positive stiffness. In Section 3, the theoretical analysis of flexural waves in Timoshenko beam without and with negative stiffness component inclusions are modelled. Section 4 provides a detailed parametric analysis of the continuous beam as a function of damping ratio of the propagating flexural waves against the frequency for various parameters of the Timoshenko beam. Section 5 presents the main conclusions of the paper.

\section{Negative stiffness component}

The idea of using negative stiffness components to suppress vibration is no longer new in engineering. Redistributing the damping over three springs as suggested by Antoniadis et al. (Antoniadis et al. 2015) is introduced here. The negative stiffness component is designed in such a way that the static stiffness is the same as that of the reference beam (Chronopoulos et al. 2015)

$$
k_{T}=k_{S}+\frac{k_{N} k_{P}}{k_{N}+k_{P}}
$$

The linear relationship between $k_{T}$ and $k_{S}$ is such that

$$
k_{S}=\alpha k_{T}
$$

In order for the negative stiffness not to cause the instability in the system, an engineering safety is introduced in such a way that 


$$
k_{S}+\frac{(1+\varepsilon) k_{N} k_{P}}{(1+\varepsilon) k_{N}+k_{P}}=0
$$

In view of Eqns (1) - (3), then:

$$
\begin{aligned}
& k_{P}=k_{T} \frac{\varepsilon \alpha(\alpha-1)}{1+\varepsilon(1-\alpha)} \\
& k_{N}=-k_{T} \frac{\varepsilon \alpha(\alpha-1)}{1+\varepsilon}
\end{aligned}
$$

The dependence of negative and positive springs on the linear relationship between $k_{T}$ and $k_{S}$ is represented in Fig. 1

It is actually a linear stable oscillator, designed in such a way that it has a static stiffness $k_{T}$ which is the same to the one of the reference SDoF oscillator. From Fig. 1, it can be seen that an increase in $\varepsilon$ will demand a higher stiffness for $k_{P}$ and $k_{N}$ respectively, in order for a definite static stiffness $k_{T}$ to be achieved.

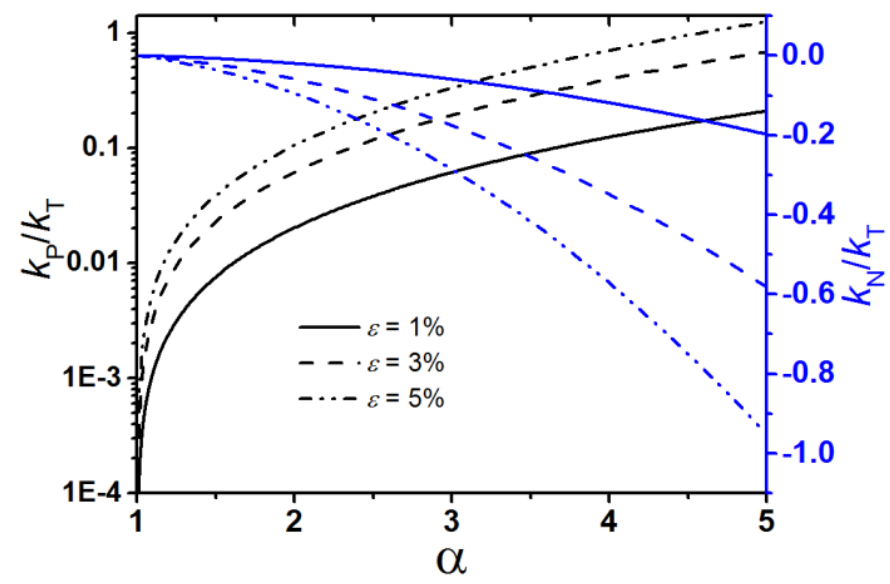

Fig. 1: The function of $\alpha$ on $k_{P} / k_{T}$ and $k_{N} / k_{T}$ for the considered system to maintain equivalent static stiffness without negative stiffness

Let us review the crux of this damping phenomenon in the presence of negative stiffness component as presented by Antoniadis et al. (2015) for a linear oscillator defined with a stiffness $k$, a mass $m$ and with a constant damping term $\eta$. From the basic mechanics, such oscillator will have $\omega_{0}^{2}=k / \mathrm{m}$ and $\xi_{0}=\eta / 2 \sqrt{\mathrm{km}}$. For this numerical example, let $\omega_{0}=2 \pi \mathrm{rad} / \mathrm{s}, k=1 \mathrm{~N} / \mathrm{m}$, and a damping factor $\eta=0.0032$. The linear oscillator works as in redistributing the static stiffness in terms of new constants defined in Eq. (1); $k_{S}, k_{P}$ and $k_{N}$. This is done to maximize the damping characteristics of the oscillator. Following the design procedure in Antoniadis et al. (2015), the relationship of $\alpha$ and damping ratio is given in Fig. 2 for various engineering values. 


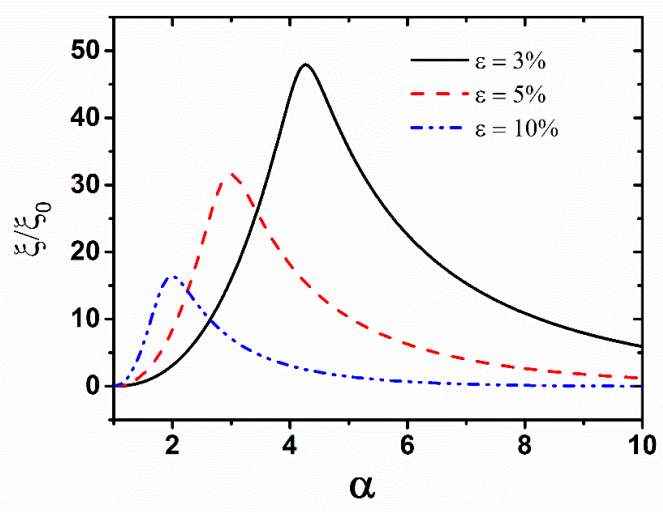

Fig. 2: The relationship of damping ratio as a function of $\alpha$ for different $\varepsilon$ parameter on the linear oscillator

Two deductions can be made from this graph; the lower the engineering safety the better the damping enhancement. In addition, the damping can be enhanced up to about 50 more than the original damping of the linear oscillator. This enhancement of the damping is investigated on Timoshenko beam in Section 3 as it has been done for Euler-Bernoulli beam (Chronopoulos et al. 2015)

\section{Negative stiffness component}

\subsection{Flexural waves in Timoshenko beam}

The governing differential equation for free flexural vibration of the Timoshenko beam shown in Fig. 1 (a) can be written as follows (Zhu et al. 2014; Zuo et al. 2016):

$$
\begin{gathered}
\kappa G A\left(\frac{\partial^{2} w}{\partial x^{2}}-\frac{\partial \theta}{\partial x}\right)-\rho A \frac{\partial^{2} w}{\partial t^{2}}-\eta \frac{\partial w}{\partial t}-k_{T} w=0 \\
E I \frac{\partial^{2} \theta}{\partial x^{2}}+\kappa G A\left(\frac{\partial w}{\partial x}-\theta\right)-\rho I\left(\frac{\partial^{2} \theta}{\partial t^{2}}\right)=0
\end{gathered}
$$

where $\rho, E$, and $G$ are the density, Young's modulus, and shear modulus respectively; $\kappa$ is the Timoshenko shear coefficient; $A$ is the cross section area; $I$ is the cross-section-area moment of inertia about the axis perpendicular to $\mathrm{x}$ and y axes. $w(x, t)$ is the transverse displacement in the $\mathrm{y}$ direction of the local coordinates, $\theta(x, t)$ is the rotation angle due to bending, $k_{T}$ is the stiffness and $\eta$ is the viscous damping element. 


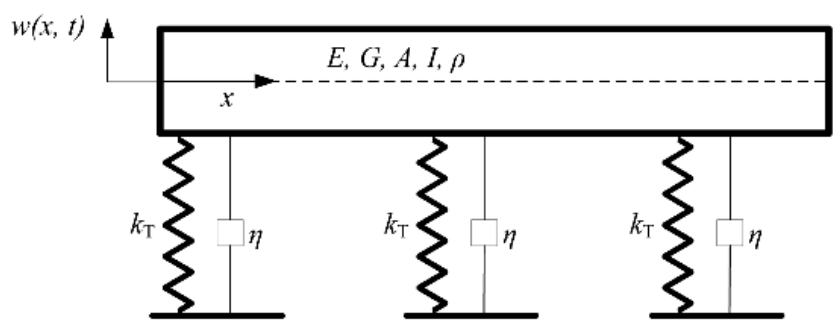

(a)

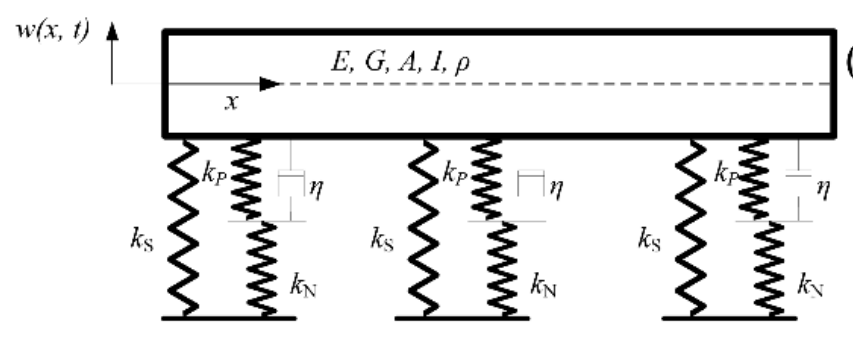

(b)

Fig. 3. A continuous Timoshenko beam (a) on a linear elastic foundation with damping (b) on a metamaterial foundation with damping.

Excluding the angle $(x, t)$, the single equation with respect to the displacement $w(x, t)$ is obtained as:

$$
\begin{aligned}
& E I w^{I V}+\rho A \ddot{w}+\eta \dot{w}-\rho I\left(1+\frac{E}{\kappa G}\right) \ddot{w}^{\prime \prime}-\frac{E I \eta}{\kappa G A} \dot{w}^{\prime \prime} \\
& +\frac{\rho I}{\kappa G A}(\rho A \dot{w}+\eta) \ddot{w}+k_{T}\left(w+\frac{\rho I}{\kappa G A} \ddot{w}-\frac{E I}{\kappa G A} w^{\prime \prime}\right)=0
\end{aligned}
$$

where prime and dot denote differentiation with respect to $x$ and $t$, respectively. Assuming time harmonic wave motion and applying Bloch's theorem [44] as:

$$
w=W e^{j k x+\lambda t}
$$

With wavenumber $\mathrm{k}$, complex frequency function $\lambda$ which allows time induced wave attenuation, wave amplitude $\mathrm{W}$, longitudinal distance $\mathrm{x}$. the frequency function consists of two parts; the real part represents the damping factor and the imaginary part the propagating waves within the structure, hence it can be expressed as

$$
\lambda(k)=-\xi(k) \omega(k) \pm i \omega_{d}(k)
$$

Inserting Eq. (10) into (8) then becomes;

$$
\begin{aligned}
& E I k^{4}+\rho A \lambda^{2}+\eta \lambda+\rho I\left(1+\frac{E}{\kappa G}\right) \lambda^{2} k^{2}+\frac{E I \eta}{\kappa G A} \lambda k^{2}+\frac{\rho I}{\kappa G A}(\rho A \lambda+\eta) \lambda^{3} \\
& +k_{T}\left(1+\frac{\rho I}{\kappa G A} \lambda^{2}-\frac{E I}{\kappa G A} k^{2}\right)=0
\end{aligned}
$$




\subsection{Flexural waves in Timoshenko beam with negative stiffness component}

The governing equations for the transverse motion of the Timoshenko beam $\mathrm{w}$ and the internal DoF y for Fig. 1 (b) can be written as:

$$
\begin{gathered}
\kappa G A\left(\frac{\partial^{2} w}{\partial x^{2}}-\frac{\partial \theta}{\partial x}\right)-\rho A \frac{\partial^{2} w}{\partial t^{2}}-\eta\left(\frac{\partial w}{\partial t}+\frac{\partial y}{\partial t}\right)-k_{p}(w-y)-k_{s} w=0 \\
E I \frac{\partial^{2} \theta}{\partial x^{2}}+\kappa G A\left(\frac{\partial w}{\partial x}-\theta\right)-\rho I\left(\frac{\partial^{2} \theta}{\partial t^{2}}\right)=0 \\
m_{c} \frac{\partial^{2} y}{\partial t^{2}}+\eta\left(\frac{\partial y}{\partial t}-\frac{\partial w}{\partial t}\right)+k_{P}(y-w)+k_{N} y=0
\end{gathered}
$$

We seek traveling wave solutions to Eqs. (12) - (14)

$$
w=W e^{-j k x+\lambda t}, y=Y e^{-j k x+\lambda t}
$$

Substituting Eq. (15) into Eq. (14) gives;

$$
Y=\frac{\left(\eta \lambda+k_{P}\right) W}{\left(\rho_{2} A_{2} \lambda^{2}+\eta \lambda+k_{N}+k_{P}\right)}
$$

Solving Eqs. (12) and (13) into a single equation, then substituting Eqn. (16) gives:

$$
\begin{aligned}
& E I k^{4}+\rho I k^{2} \lambda^{2}\left(1+\frac{E}{\kappa G}\right)+\frac{E I \eta}{\kappa G A} \lambda k^{2}(1+\Delta)+M \lambda^{2}+k^{2} \frac{k_{p} E I}{\kappa G A}(1-\Delta)+\frac{k_{S} E I}{\kappa G A} k^{2} \\
& +\eta \lambda(1+\Delta)+k_{p}(1-\Delta)+k_{s}+\frac{\rho^{2} I}{\kappa G} \lambda^{4}+\frac{\eta \rho I}{\kappa G A} \lambda^{3}(1+\Delta)+\frac{k_{p} \rho I}{\kappa G A} \lambda^{2}(1-\Delta)+\lambda^{2} \frac{k_{s} \rho I}{\kappa G A}=0
\end{aligned}
$$

where $\Delta=\frac{\left(\eta \lambda+k_{P}\right)}{\left(m \lambda^{2}+\eta \lambda+k_{N}+k_{P}\right)}$.

\section{Parametric case studies}

To investigate the damping characteristics of Timoshenko beam on the damping ratio, we set the physical constants $E=700 M P a, \rho=7850 \mathrm{~kg} / \mathrm{m}^{3}, M=\rho A, v=0.3, I=10^{-5} \mathrm{~m}^{4}, A=$ $1 \mathrm{~m}^{2}, \eta=0.1, \kappa=0.9$ and $k_{T}=10^{7} \mathrm{~N} / \mathrm{m}$.

\subsection{Effects of Shear on frequency and damping ratio}

To quantify the effects of shear on the wavenumber and frequency for the system when $m_{c}=0$ are shown in Fig 4 (a) and 5(a). There are two observations that can be noticed in these two figures; both configurations (elastic and metamaterial foundations) are statically and dynamically the same. This is seen in the plot of wavenumber and frequency. Secondly, it can be observed that the shear increase tends to increase the bandgap for the system. For illustration, when shear $\kappa=0.1 \mathrm{e}$, frequency band gap is observed for the flexural wave between $0-111 \mathrm{~Hz}$ while when $\kappa=0.9$, the frequency stopband is $0-331 \mathrm{~Hz}$.

The damping ratio effect on the flexural wave for the beam on elastic and metamaterial foundation are presented in Fig 4 (b) and 5 (b). Apart from the bandgap zone that is also shown in the damping frequency plots, we noticed that there is an extraordinary increase of the damping ratio for the system having metamaterial foundation compared to the elastic foundation. In addition, the 
damping ratio $\xi$ for the flexural wave is frequency dependents for metamaterial foundations as against constant value for the elastic foundation for all frequencies. An increase in shear also causes an increase in the damping ratio of the Timoshenko beam on metamaterial foundation. There is also a sharp convergence to the elastic foundation damping ratio when $\kappa=0.1$ compared to when $\kappa=0.9$. Therefore, having a high shear values is more beneficial in increasing the damping ratio of flexural waves in Timoshenko beams.
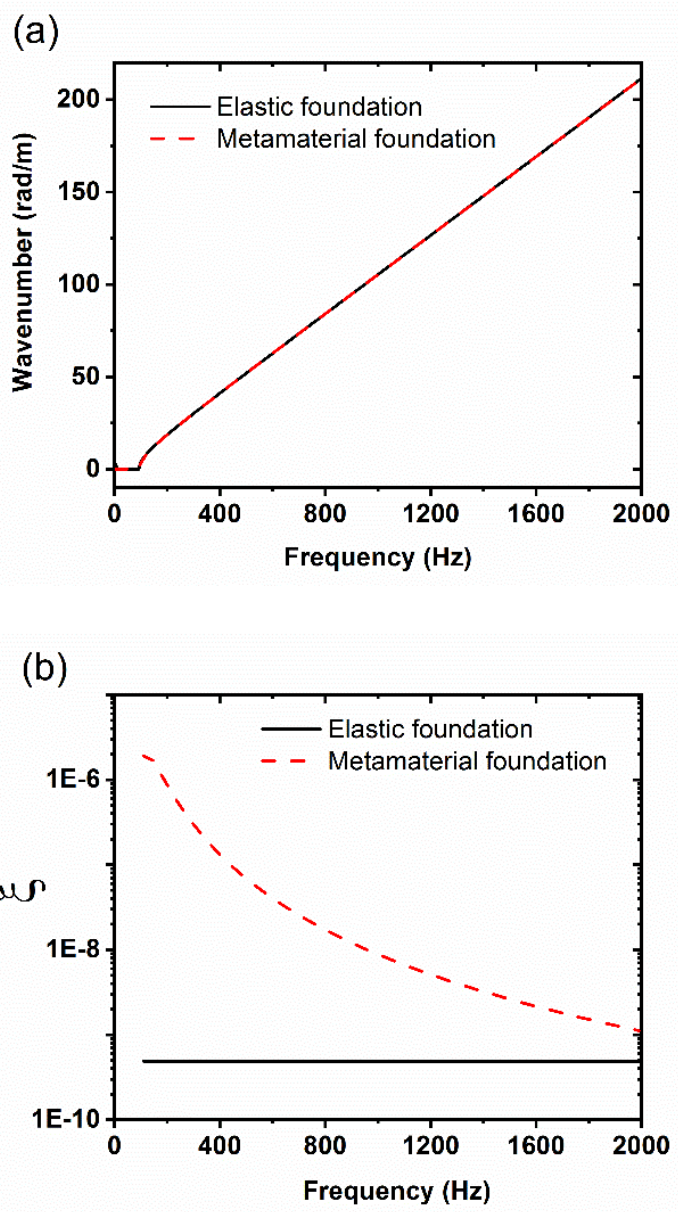

Fig 4. (a) Dispersion curve for beam on elastic foundation and metamaterial (b) Damping ratio $\xi$ as a function of frequency for beam elastic foundation and metamaterial. Computations conducted with $\alpha=1.6, \kappa=0.1, \eta=0.1$ 

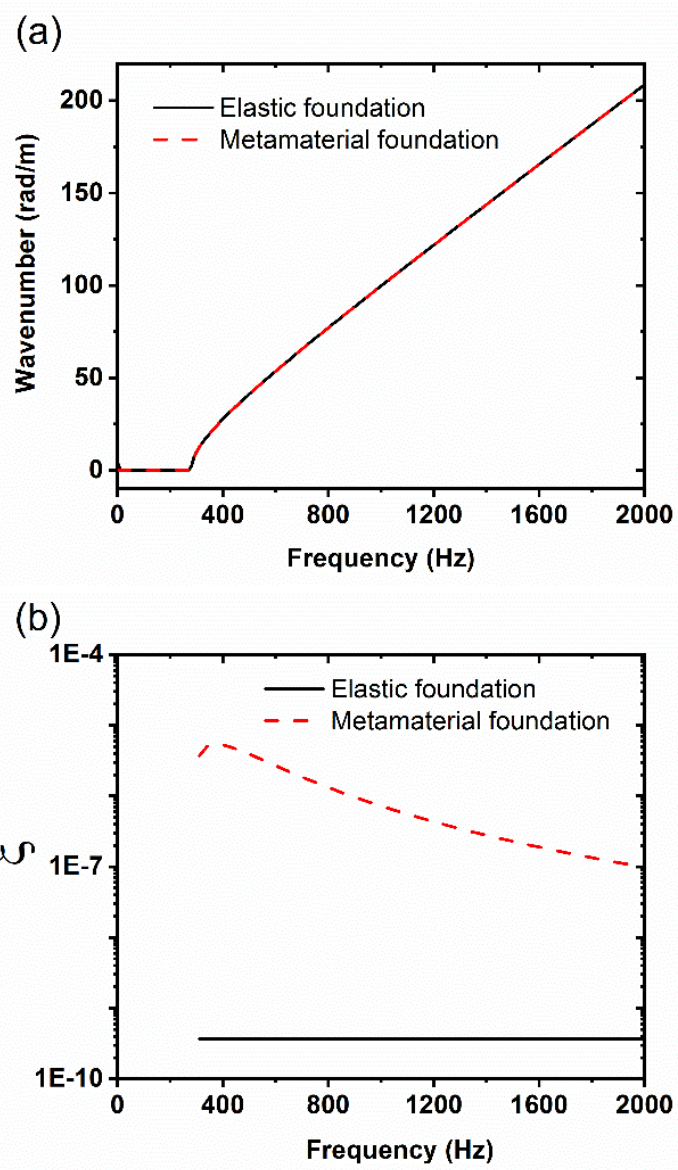

Fig 5. (a) Dispersion curve for beam on elastic foundation and metamaterial (b) Damping ratio $\xi$ as a function of frequency for beam elastic foundation and metamaterial. Computations conducted with $\alpha=1.6, \kappa=0.9, \eta=0.1$

\subsection{Effects of Alpha on damping ratio}

Figure 6 presents the damping ratio of the system with metamaterial foundation for various values of $\alpha$. When the relationship between $k_{T}$ and $k_{S}$ is 1.2, the damping ratio gives higher values than that of $\alpha$ equals 1.4 and 2.5. It should be stated clearly that there is an optimal value of $\alpha$ to give the highest damping for the system as presented in Fig. 2, and in Antoniadis et al. (2015). 


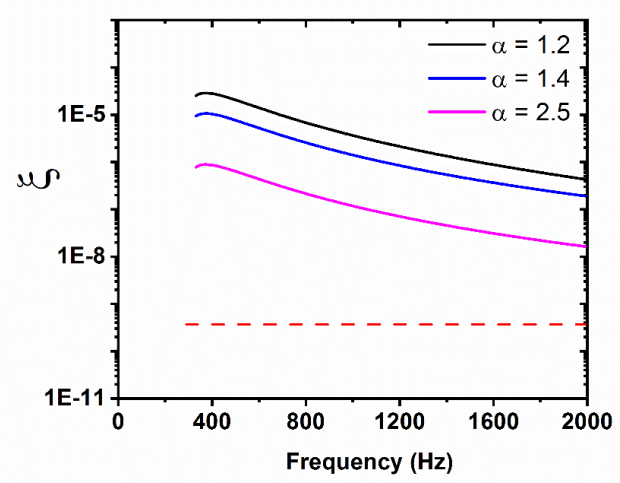

Fig 6. Damping ratio $\xi$ for the Timoshenko beam on metamaterial foundation. Computations conducted with $\kappa=0.9, \eta=0.1, \varepsilon=3 \%$

\subsection{Effects of engineering safety}

Engineering safety has a significant effect of the damping ratio of the flexural waves as can be seen in Fig. 7. Increment in engineering safety simultaneously increases negative and positive springs in the system. The magnitude of the damping ratio is high when the engineering safety is low. As a consequence, the presence of small value of negative stiffness component in the system makes for the hyper damping characteristics of the flexural waves in the beam. As expected for the elastic foundation, the damping ratio is constant for all the frequencies.

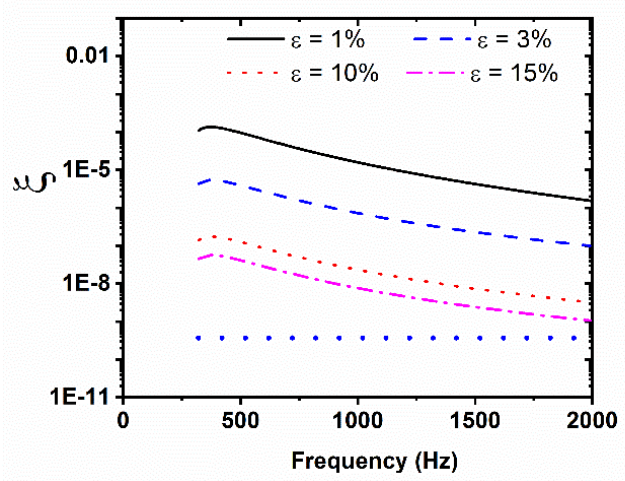

Fig 7. Damping ratio $\xi$ for the flexural wave propagating on metamaterial foundation.

Computations conducted with $\alpha=1.6$

\subsection{Effects of damping coefficient on the damping ratio}

The damping ratio versus frequency plot for three damping coefficients is presented in Fig. 8. The dependent of the damping ratio for the metamaterial foundation is also observed here. An increase in damping coefficient causes increase in the damping ratio for the flexural waves in the Timoshenko beam. However, the increase in damping coefficient is not proportional to the increase in damping ratio. We can see that for the metamaterial foundation model, the damping ratio is converging towards the elastic foundation damping ratio. 


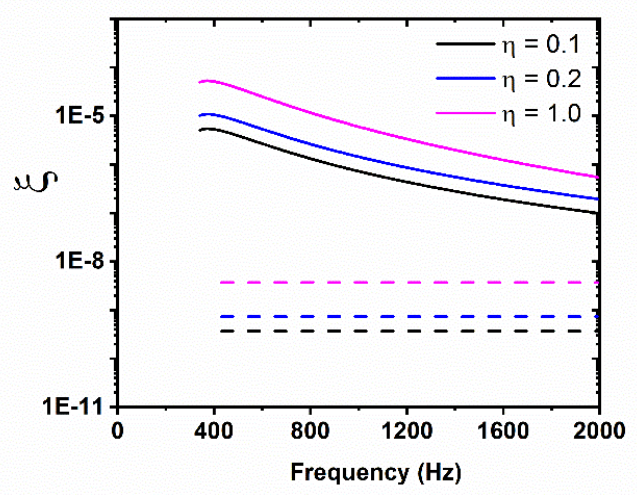

Fig 8. Damping ratio $\xi$ for the flexural wave propagating on metamaterial foundation.

Computations conducted with $\alpha=1.6, \varepsilon=3 \%$

\subsection{Effects of resonating mass greater than zero}

When the resonating mass of the metamaterial foundation is not zero as we previously saw, it will affect the damping ratio for the system. The effect of resonating mass is shown in Fig. 9. It is observed that at low frequency region, the damping ratios when $m_{c}>0$ are higher than when $m_{c}=0$. As much as the negative stiffness component helps in improving the damping properties of the flexural wave, the resonating mass can also be adequately designed to maximize damping ratio within a frequency range.

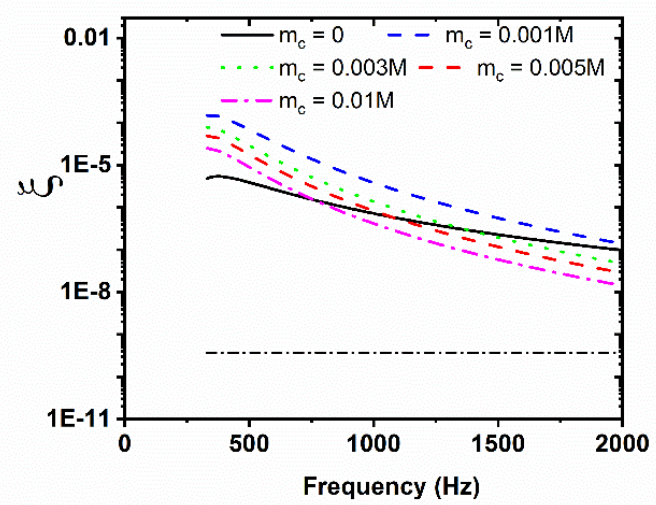

Fig 9. Damping ratio $\xi$ for the flexural wave propagating on metamaterial foundation.

Computations conducted with $\alpha=1.6, \varepsilon=3 \%$

\section{Conclusion}

In this paper, we investigated flexural wave propagation in Timoshenko beam with elastic and metamaterial foundations. The metamaterial foundation was formed with an elastic constant and negative stiffness connection and by the reallocation of the damping element. This paper has revealed how a continuous structure can be connected to a metamaterial foundation to increase 
the magnitude of the damping in the system. The wave phase for Timoshenko beam connected to elastic and metamaterial beams was found to be equivalent; however, the damping ratio was quite different for the considered frequency range. The parametric study also revealed that shear coefficient, engineering safety and resonating mass had significant effects on the damping ratio of the continuous beam. And these properties can be designed in such a way to maximise the hyper damping properties of the flexural waves propagating within the beam.

\section{References}

Abohadima, S., Taha, M., \& Abdeen, M. A. M. (2015). General Analysis of Timoshenko Beams on Elastic Foundation, Mathematical Problems in Engineering, 1-11.

Antoniadis I, Chronopoulos D, Spitas V., \& Koulocheris D. (2015). Hyper-damping properties of a stiff and stable linear oscillator with a negative stiffness element, Journal of Sound and Vibration, 346, 37-52.

Abbas B. A. H, Thomas J. (1978). Dynamic stability of Timoshenko beams on an elastic foundation, Journal of Sound and Vibration, 60(1), 33-34.

Capsoni A, Capsoni A, Maria G, \& Bani-hani K. (2013). On damping effects in Timoshenko beams International Journal of Mechanical Sciences On damping effects in Timoshenko beams, International Journal of Mechanical Sciences, 73, 27-39.

Chronopoulos D, Antoniadis I, Collet M, \& Ichchou M. (2015). Enhancement of wave damping within metamaterials having embedded negative stiffness inclusions, Wave Motion, 58, 165179.

Haldar S, Basu D. (2013). Response of Euler-Bernoulli beam on spatially random elastic soil, Computers and Geotechnics, 30, 110-128.

Kahrobaiyan M. H, Asghari M, \& Ahmadian M. T. (2014). A Timoshenko beam element based on the modified couple stress theory, International Journal of Mechanical Sciences, 79, 7583.

Lee S. Y, Kuo Y. H, \& Lin F. Y. (1992). Stability of a Timoshenko beam resting on a Winkler elastic foundation, Journal of Sound and Vibration, 153(2), 193-202.

Liu X., Shi Z, \& Mo Y. L. (2016). Effect of initial stress on periodic Timoshenko beams resting on an elastic foundation, Journal of Vibration and Control, 1-14.

Rivin E. I. (2010). Passive Vibration Isolation Means. In Passive Vibration Isolation.

Wang X. Q, So R. M. C. (2015). Timoshenko beam theory: A perspective based on the wavemechanics approach, Wave Motion, 57, 64-87.

Yu D., Liu Y, Wang G., Zhao H, Qiu, J. (2006). Flexural vibration band gaps in Timoshenko beams with locally resonant structures, Journal of Applied Physics, 100(12).

Zhang H. (2019). Free Vibration of Timoshenko Beams on Elastic Foundation with Horizontal Frictions Free Vibration of Timoshenko Beams on Elastic Foundation with Horizontal Frictions, Journal of Vibration Engineering \& Technologies, 2(3), 305-314.

Zhou J, Wang K, Xu D, Ouyang H. (2017). Multi-low-frequency flexural wave attenuation in Euler-Bernoulli beams using local resonators containing negative-stiffness mechanisms, Physics Letters A,

Zhu R, Liu X. N, Hu G. K, Sun C. T, Huang, G. L. (2014). A chiral elastic metamaterial beam for broadband vibration suppression, Journal of Sound and Vibration, 333(10), 2759-2773.

Zuo S. L, Li F. M, Zhang, C. (2016). Numerical and experimental investigations on the vibration band-gap properties of periodic rigid frame structures, Acta Mechanica, 227(6), 1653-1669. 